\title{
Resolving the Issues of Capon and APES Approach for Projecting Enhanced Spectral Estimation
}

\author{
Kantipudi MVV Prasad*, Dr. H.N. Suresh** \\ * Visvesvaraya Technological University, Belgaum, India \\ ** Department of Instrumentation Bangalore Institute Technology, Bangalore, India
}

\begin{abstract}
Article Info
Article history:

Received Sep 15, 2015

Revised Nov 30, 2015

Accepted Dec 16, 2015

\section{Keyword:}

APES

Capon Estimators

Power Spectral Density

Spectral Estimation

ABSTRACT

There are various applications on signal processing that is highly dependent on preciseness and accuracy of the outcomes in spectrum of signals. Hence, from the past two decades the research community has recognized the benefits, significance, as well as associated problems in carrying out a model for spectral estimation. While in-depth investigation of the existing literatures shows that there are various attempts by the researchers to solve the issues associated with spectral estimations, where majority of teh research work is inclined towards addressing problems associated with Capon and APES techniques of spectral analysis. Therefore, this paper introduces a very simple technique towards resolving the issues of Capon and APES techniques. The outcome of the study was analyzed using correlational factor and power spectral density to find the proposed system offers better spectral estimations compared to existing system.
\end{abstract}

Copyright $\left({ }^{\circ} 2016\right.$ Institute of Advanced Engineering and Science. All rights reserved.

\section{Corresponding Author:}

Kantipudi MVV Prasad

Research Scholar

Visvesvaraya Technological University, Belgaum, India

E-Mail: prasadb2016@gmail.com

\section{INTRODUCTION}

In the area of signal processing, the spectral estimation plays a critical role for many applications. Some of the effective examples for this purpose can be said to be target of identifying the periodicity of a signal in time-series, which can be stated as a problem of spectral estimation [1]. The conventional techniques to perform spectral estimations adopt Discrete Fourier Transform (DFT) and its various versions. There are various researchers that have attempted to introduce various parametric techniques with enhanced resolution approach for achieving better potential in resolving issues of spectral estimations [2] [3]. Some of the recent efficient approaches that come under the non-parametric trends are conventional Capon estimators and Amplitude and Phase Estimation (APES) techniques [4]. However, a closer look into Capon estimators and Amplitude and Phase Estimation (APES) techniques shows that they exhibit performance equivalent to conventional DFT approaches under certain conditions. In this paper, we emphasize the utilities of spectral estimation techniques as they are frequently used in many major applications of signal processing e.g. designing control systems, bio-medical signal analysis, processing speech, and finding latent periodicity. These estimation techniques are more or less inclined towards time factor while analysis a spectrum. There are also applications found for spectral estimations considering the spatial factors e.g. localizing the source using wireless sensors. We also emphasize on the unsolved problem associated with the spectral estimation techniques that calls for evaluation of the cumulative power that are being distributed over certain range of frequency from a fixed record of static sequences of data. This paper has presented a very simple technique to enhance the capabilities of spectral estimation techniques considering the case study of Capon and APES estimation process. The paper will also show significant improvement of power spectral density as well as correlational factor by introducing the proposed technique of spectral analysis. Section 2 discusses about the 
research methodology and research and discussion has discussed in Section 3. Finally, Section 4 makes some concluding remarks and highlights the direction of future work to be carried out.

\subsection{Background}

This section will present various significant research works introduced by the researchers most recent for addressing the problems associated with spectral estimations. Nielson et al. [5] have focused on the issues associated with Capon estimators and have mechanized a way using a variable length of new filter design for enhancing the performing of spectral estimations. The outcome of the study was analyzed using mean squared error. Studies toward Capon along with the APES estimators was also witnessed in the literature of Alty et al. [6], where the authors have adopted sliding window based on temporal factor. However, few attempts of benchmarking were found in the study. Similar pattern of study was also witnessed in the literature of Angelopoulos et al. [7]. The major emphasis of the study was to introduce the significance of coherence spectrum and to scale down the hypothetical complexities associated with Capon and APES. The study has also performed an extensive analysis of the computational complexities with respect to the length of the filter banks. The study was also found to miss out comparative analysis to showcase the effectiveness of the outcomes. Literatures have also presence of certain unique attempts to perform spectral estimation. Adoption of Gohberg factorization technique was seen in the literature of Xue et al. [8], where the authors have used weighted least squared approach of iterative nature. The outcome of the study was analyzed using computational time as well as signal power, but the outcome of the study was not found to analyze with respect to the power spectral density, which is also one of the significant performance parameters to showcase the effectiveness of spectral estimation techniques. Tezel and Yildirum [9] have focused on the problems associated with APES technique and hence the authors have introduced an adaptive impulse filtering technique considering the case study of signals being generated from the radar imaging.

Most recently, two dimensional vibration signals and their generated spectrum have being analyzed by Dan et al. [10] using damped Capon estimators. The authors have presented a spectrum representation with respect to frequency and decomposed parameters of plane of the random vibration of the real-world. The study has adopted a maximized resolution of phase and amplitude for analyzing both decomposed factor and frequency. The outcome of the study was analyzed in presented of noise to show optimal resolution of the frequency. The authors have also commented that their techniques are more applicable on extracting spectrum with precise amplitude. Sridhar and Srinivasulu [11] have adopted enhanced recursive least square technique for achieving enhanced spectral resolution. Kalagnomos et al. [12] have integrated conventional APES and its enhanced version is called as parameterized APES for enhance spectral estimations over radar imaging. Touze et al. [13] have introduced a three dimensional estimators using Capon and MUSICAL algorithm with double precision. Candes et al. [14] have designed a soft thresholding approach for performing spectral estimation using singular value over medical images for achieving an optimal spectral estimation technique.

\subsection{The Problem}

In the area of signal processing, spectral estimation plays a critical role in various applications e.g. analysis of radar signal, speech processing, and biological processing of signals etc that mainly fall under the category of time-frequency signal analysis. It was also seen that Fourier Transformation technique is mainly adopted for the purpose of evaluating spectrum of non-parametric type. Most recently, there is an increasing focus on investigating the possible use of Capon estimation techniques and adaptive amplitude and phase estimation techniques (APES). One of the significant advantages of adoption of such method using Capon and APES over conventional spectral estimation technique is enhanced resolution as well as smoother features of the signals. However, such techniques also have certain pitfalls e.g.

- Capon and APES techniques used in existing system have more dependency on computational and processing power

- It was also found that Capon and APES techniques used can render a significant amount of instability in the system.

- The peak locations found in the spectral analysis using APES techniques are usually biased accompanied by lower resolution.

Hence, the problem statement of the proposed study is - "It is a computationally challenging task to design an integrated spectral analysis considering both Capon and APES approach to ensure cost-effective computational analysis for two dimensional spectrums." 


\subsection{The Proposed Solution}

The main purpose of the proposed system is to evolve up with a technique that can introduce a spectral estimation techniques using non-parametric approach. The proposed system uses both capon and APES estimation technique to investigate the problems associated with it and provides a solution to resolve the problems. Generally, it is known that such technique works on the principle of lower variance with response of lesser deformation for ensuring optimal resolution. The spectrum generated by Capon can be considered to be a set of filters, where each of the filters is positioned in center of each other in the evaluation of frequencies. There is a higher dependencies of both frequency and data in its bandpass filters that separates Capon techniques of spectral estimations from other conventional techniques e.g. periodogram that is potentially independent of frequency as well as data using discrete matrix of Fourier Transform. Therefore, the primary goal of this manuscript is to resolve the issues of Capon and APES estimators by investigating the dynamic spectrum formulated for showing the significant relationship between any two signals. We define a term called as correlational factor to investigate the possible relationship between the two realvalued signals. The proposed system also targets to perform comparative analysis of spectral estimation with respect to existing techniques of Periodogram, PWelch, and Multitaper method considering Power Spectral Density and correlational factor.

\section{RESEARCH METHODOLOGY}

The baseline of estimation techniques for Capon approach uses filter-bank decomposition techniques. Each band involved in the process calls for its signals to be estimated. Consider an input static arbitrary process of zero-mean that will act as an input for the filters. Hence, the function representing filter can be now represented as,

$$
\mathrm{Mk}=\left[\mathrm{m}_{\mathrm{k}, 0}, \mathrm{~m}_{\mathrm{k}, 1} \ldots \mathrm{m}_{\mathrm{k}, \mathrm{s}-1}\right]^{\alpha}
$$

In the above eq. (1), Mk can be considered as function for the $k$ number of filters of size $s$. The variable $\alpha$ will represent transposition matrix. Therefore, the anticipated power factor of the output signal can be mathematically represented as,

$$
E\left\{\left|b_{k}(n)\right|^{2}\right\}=E\left\{\left|m_{k}^{\beta} X(n)\right|^{2}\right\}=m_{k}^{\beta} \cdot C_{x x . m_{k}}
$$

In the above equation (2), the variable $E$ can be termed as anticipated power of the output signal and the variable $\beta$ can be termed as conjugate transpose matrix. The covariance matrix is represented by $\mathrm{C}_{\mathrm{xx}}$ associated with the input signal $a_{n}$, it can be further written as,

$$
C_{x x}=E\left\{X(n) \cdot X^{\beta}(n)\right\}
$$

In the equation (2) and (3), the variable $\mathrm{X}(\mathrm{n})$ can be defined as a transposed matrix with an elements of $x(n)$, $x(n-1), \ldots, x(n-s+1)$. Therefore, the system can shape up the Fourier matrix $(\gamma)$ considering $\mathrm{s} \mathrm{K}$,

$$
\gamma=\left[\gamma_{0}, \gamma_{1} \ldots \gamma_{\mathrm{K}-1}\right]
$$

In the above equation (4), the variable $\gamma_{\mathrm{k}}$ is equivalent to,

$$
\frac{1}{\sqrt{s}}\left[1 \quad \operatorname { e x p } \left(j \varphi_{k} \quad \ldots \quad \exp \left(j \varphi_{k}(s-1)\right]^{\alpha}\right.\right.
$$

In the above equation (5), $\varphi_{\mathrm{k}}$ is equivalent to $2 \pi \mathrm{k} / \mathrm{K}$ ( $\mathrm{K}=$ maximum numbers of filters). Therefore, when the value of $\mathrm{K}$ as well as $s$ is equivalent, than the matrix $\mathrm{F}$ can be termed as Fourier matrix. Hence, $\mathrm{F}^{\beta}$. $\mathrm{F}=\mathrm{F}$. $\mathrm{F}^{\beta}=\mathrm{I}$ (unitary matrix). Therefore, in the Capon estimators, the system chooses the coefficients of filter for the purpose of reducing the variance of the output filter by considering the constraint factor,

$$
=m_{k}^{\beta} C_{x x \cdot m_{k}}+\lambda\left[1-m_{k}^{\beta} \cdot \gamma_{k}\right]
$$

In the above equation (6), the variable $\lambda$ can be termed as Lagrangian multiplier. Therefore, according to this constraint factor, when the input of static arbitrary process $a(n)$ is subjected to the filter $m_{k}$ with zero distortion at a particular frequency $\varphi_{k}$ as well as signals for other frequency than the baseline $\varphi_{k}$ will tend to 
be attenuated. Hence, the objective function for the purpose of reducing the constraints exhibited in equation (6) will result in,

$$
m_{k}=\frac{C_{x x}^{-1} \gamma_{k}}{\gamma_{k}^{\beta} C_{x x}^{-1} \gamma_{k}}
$$

Therefore, the mathematical representation of the spectrum can be exhibited as,

$$
\sigma_{x x}\left(\varphi_{k}\right)=E\left\{\left|b_{k}(n)\right|^{2}\right\}=m_{k}^{\beta} C_{x x . m_{k}}
$$

Substituting eq. (7) to eq. (8),

$$
\sigma_{x x}\left(\varphi_{k}\right)=\frac{1}{\gamma_{k}^{\beta} \cdot C_{x x}^{-1} \cdot \gamma_{k}}
$$

Therefore, $C_{x x . m_{k}}=\sigma_{x x}\left(\varphi_{k}\right) \cdot \gamma_{k}$

The above equation (10) can be now generalized by considering $\gamma_{\mathrm{k}}$, where $\mathrm{k}=0,1,2,3 \ldots \mathrm{K}-1$,

$$
\mathrm{C}_{\mathrm{xx}} \mathrm{M}=\gamma \cdot \sigma_{\mathrm{xx}}(\varphi)
$$

In the above equation (11), the variable $M$ refers to a matrix with elements $m_{0}, m_{1}, m_{2} \ldots m_{K-1}$. The function $\sigma_{\mathrm{xx}}(\varphi)$ will refer to the diagonal elements of $\sigma_{\mathrm{xx}}\left(\varphi_{0}\right), \sigma_{\mathrm{xx}}\left(\varphi_{1}\right) \ldots \sigma_{\mathrm{xx}}\left(\varphi_{\mathrm{K}-1}\right)$. The design of the proposed system is being carried out on Matlab on normal 32 bit machine. For precise evaluation of the proposed system, the implementation is being carried out to evaluate the analysis of cross-spectrum and thereby establish the relationship between two signals for enhanced spectral estimations. Generally, the cross-spectrum is used to accomplish the lag in phases between sinusoidal components of the signal. Hence, it is essential to recognize the potential correlational factor at specific frequencies. The proposed system adopts the term Correlational factor for identifying the potential frequency domain between the signals. In this case the proposed system considers the presence of two input signals of arbitrary type $a_{1}(n)$ and $a_{2}(n)$ associated with spectrum $\sigma_{a 1 a 1}$ $\left(\varphi_{\mathrm{k}}\right)$ and $\sigma_{\mathrm{a} 2 \mathrm{a} 2}\left(\varphi_{\mathrm{k}}\right)$. Therefore, the designs of the filter can be represented as,

$$
m_{p, k}=\frac{C_{a_{p} a_{p}}^{-1} \cdot \gamma_{k}}{\gamma_{k}^{\beta} C_{a_{p} a_{p}}^{-1} \gamma_{k}}
$$

In the above equation (12), the variable $\mathrm{p}$ represents values worth 1,2 . The above design of the filter can be utilized for estimating the spectrum of $a_{1}(n)$ and $a_{2}(n)$ at a specific frequency of $\varphi_{k}$.

$$
\sigma_{a_{p} a_{p}}\left(\varphi_{k}\right)=\frac{1}{\gamma_{k}^{\beta} C_{a_{p} a_{p}}^{-1} \gamma_{k}}
$$

In the above equation, the matrix for covariance corresponding to a particular signal $a_{p}(n)$ can be now represented as,

$$
C_{a_{p} a_{p}}=E\left\{X_{p}(n) X_{p}^{\beta}(n)\right\}
$$

The mathematical representation of the signal $a_{p}(n)$ can be shown as,

$$
X_{p}(n)=\left[\begin{array}{llll}
X_{p}(n) & x_{p}(n-1) & \ldots & x_{p}(n-s+1)
\end{array}\right]^{T}
$$

The mathematical representation of the cross-spectrum for two input arbitrary process $a_{1}(n)$ and $a_{2}(n)$ is,

$$
\sigma_{a_{1} a_{2}}\left(\varphi_{k}\right)=E\left\{b_{2, k(n)} b_{1, k(n)}^{*}\right\}
$$




$$
\sigma_{a_{2} a_{1}}\left(\varphi_{k}\right)=E\left\{b_{2, k(n)} b_{1, k(n)}^{*}\right\}=\sigma_{a_{1} a_{2}}^{*}\left(\varphi_{k}\right)
$$

In the above equation (16) and (17), $\mathrm{b} 1, \mathrm{k}_{(\mathrm{n})}$ and $\mathrm{b}_{2, \mathrm{k}(\mathrm{n})}$ is considered to be output for filters $\mathrm{m}_{1, \mathrm{k}}$ and $\mathrm{m}_{2 \mathrm{k}}$ respectively. The symbol $*$ in equation $(16)$ represents operator for conjugate complex. Normalizing equation (16),

$$
\sigma_{a_{1} a_{2}}\left(\varphi_{k}\right)=m_{1, k}^{\beta} C_{a_{1} a_{2}} m_{2, k}
$$

In the above equation (18), the matrix for cross-correlation for input process a1 (n) and a2 (n) can be now represented as,

$$
C_{a_{1} a_{2}}\left(\varphi_{k}\right)=E\left\{X_{1}(n) \cdot X_{2}^{\beta}(n)\right\}
$$

Therefore, substitution (12) in (18)

$$
\sigma_{a_{1} a_{2}}\left(\varphi_{k}\right)=\frac{\gamma_{k}^{\beta} C_{a_{1} a_{1}}^{-1} C_{a_{1} a_{2}} C_{a_{2} a_{2}}^{-1} \cdot \gamma_{k}}{\left[\gamma_{k}^{\beta} C_{a_{1} a_{1}}^{-1} \gamma_{k}\right]\left[\gamma_{k}^{\beta} C_{a_{2} a_{2}}^{-1} \gamma_{k}\right]}
$$

Hence, this equation can be used for spectral estimation for cross-spectrum in signal processing. In the next phase of the evaluation, the proposed system will be focused on establishing the correlational factor between the two static input process of arbitrary type $a_{1}(n)$ and $a_{2}(n)$. Hence, the proposed system represents the correlational factor mathematically as,

$$
\theta_{a_{1} a_{2}}^{2}\left(\varphi_{k}\right)=\frac{\left|\sigma_{a_{1} a_{2}}\left(\varphi_{k}\right)\right|^{2}}{\sigma_{a_{1} a_{1}}\left(\varphi_{k}\right) \sigma_{a_{2} a_{2}}\left(\varphi_{k}\right)}
$$

The similar estimations for the cross-correlational factor for equation (20) will be,

$$
\left|\sigma_{a_{1} a_{2}}\left(\varphi_{k}\right)\right|^{2}=\frac{\left[\gamma_{k}^{\beta} C_{a_{1} a_{1}}^{-1} C_{a_{1} a_{2}} C_{a_{2} a_{2}}^{-1} \cdot \gamma_{k}\right]^{2}}{\left[\gamma_{k}^{\beta} C_{a_{1} a_{1}}^{-1} \gamma_{k}\right]^{2}\left[\gamma_{k}^{\beta} C_{a_{2} a_{2}}^{-1} \gamma_{k}\right]^{2}}
$$

Substitution and normalizing of equation (13) and (22) on equation (21)

$$
\theta_{a_{1} a_{s}}^{2}\left(\varphi_{k}\right)=\frac{\left[\gamma_{k}^{\beta} C_{a_{1} a_{1}}^{-1} C_{a_{1} a_{2}} C_{a_{2} a_{2}}^{-1} \cdot \gamma_{k}\right]^{2}}{\left[\gamma_{k}^{\beta} C_{a_{1} a_{1}}^{-1} \gamma_{k}\right]^{2}\left[\gamma_{k}^{\beta} C_{a_{2} a_{2}}^{-1} \gamma_{k}\right]^{2}}
$$

\section{RESULT AND DISCUSSION}

The outcome of the proposed system is analyzed on multiple scenarios. Figure 1 shows the outcome of the existing method of correlational factor, where the Matlab function mscohere is used to estimate the magnitude squared coherence factor. The existing method of spectral estimation is found to use on Welch approach based on periodogram technique. 


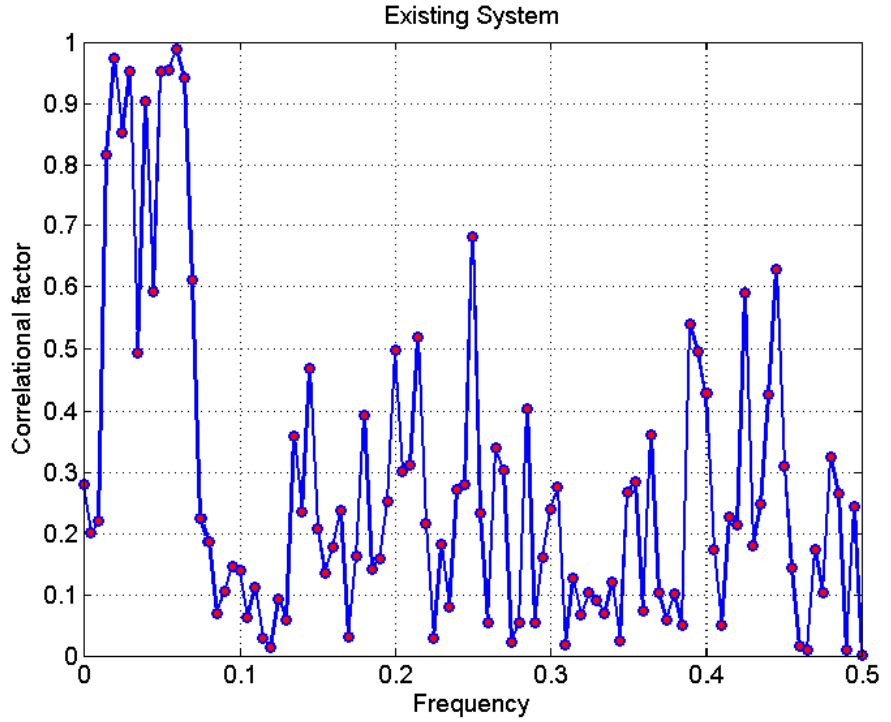

Figure 1. Correlational Factor of Existing System

The outcome of the study considers the same input variables of $a_{1}(n)$ and $a_{2}(n)$ considering the set of frequencies $\mathrm{f}_{0}, \mathrm{f}_{1}, \ldots, \mathrm{f}_{\mathrm{N}-1}$. The mathematical representation of the signals can be done as,

$$
\begin{aligned}
& a_{1}(n)=\varphi_{1}(n)+\sum_{i=0}^{N-1} \cos \left(2 \pi f_{i} n\right) \\
& a_{s}(n)=\varphi_{2}(n)+\sum_{i=0}^{N-1} \cos \left[2 \pi\left(f_{i} n+\delta_{i}\right]\right.
\end{aligned}
$$

In the above equations, the system considers $\varphi_{1}(\mathrm{n})$ and $\varphi_{2}(\mathrm{n})$ as the Gaussian arbitrary process with variance of 1 . The system also considers the phase shifts of $\delta_{0}, \delta_{1}, \ldots, \delta_{\mathrm{N}-1}$ with arbitrary assumptions considering 1024 samples (n).

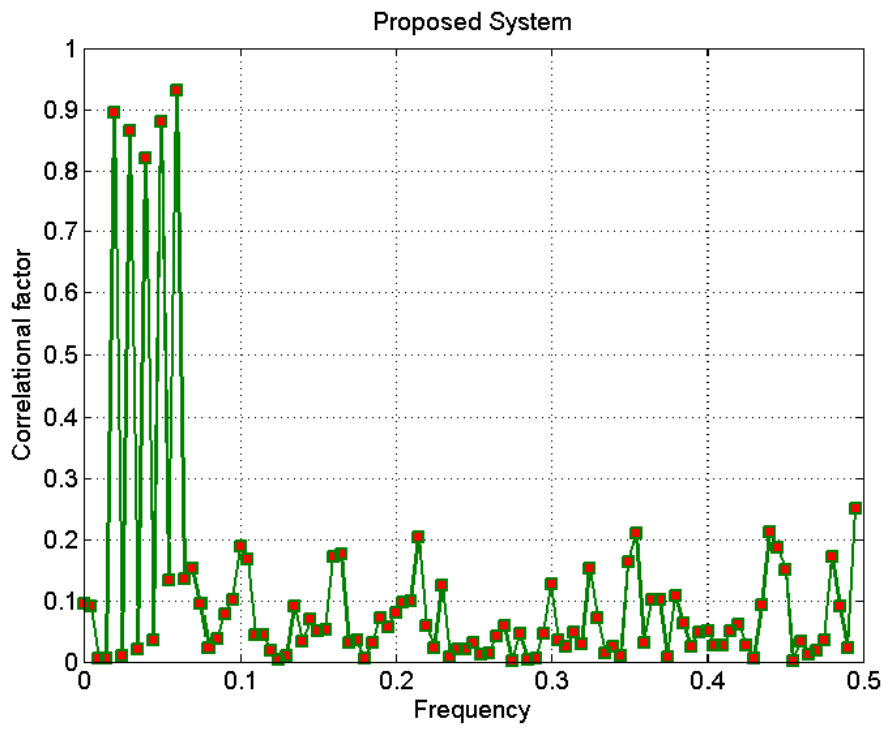

Figure 2. Correlational Factor of Proposed System 
Figure 2 shows the outcome for correlation factor for the proposed mechanism of spectral estimation. The system considers the windows length of 100-200 with samples of frequencies as $\mathrm{f}_{0}=0.02$, $\mathrm{f}_{1}=0.03, \mathrm{f}_{2}=0.04, \mathrm{f}_{3}=0.05, \mathrm{f}_{4}=0.06$. The markers are given intentionally in the analysis to have a clear visualization of the spectrum being generated. Hence, the correlational factor for the proposed system is found to have significant peaks as compared to the existing system. In order to further perform comparative analysis, we also extend the simulation study considering all the major existing system e.g. Periodogram, PWelch, and Multitaper method with the proposed mechanism of spectrum estimations using capon and APES method. The outcomes of all these methods are exhibited in Figure 3-7, where the analysis is done with respect to Power Spectral Density (PSD) for better visualization of the spectrum being generated by all the individual methods of spectrum estimations.

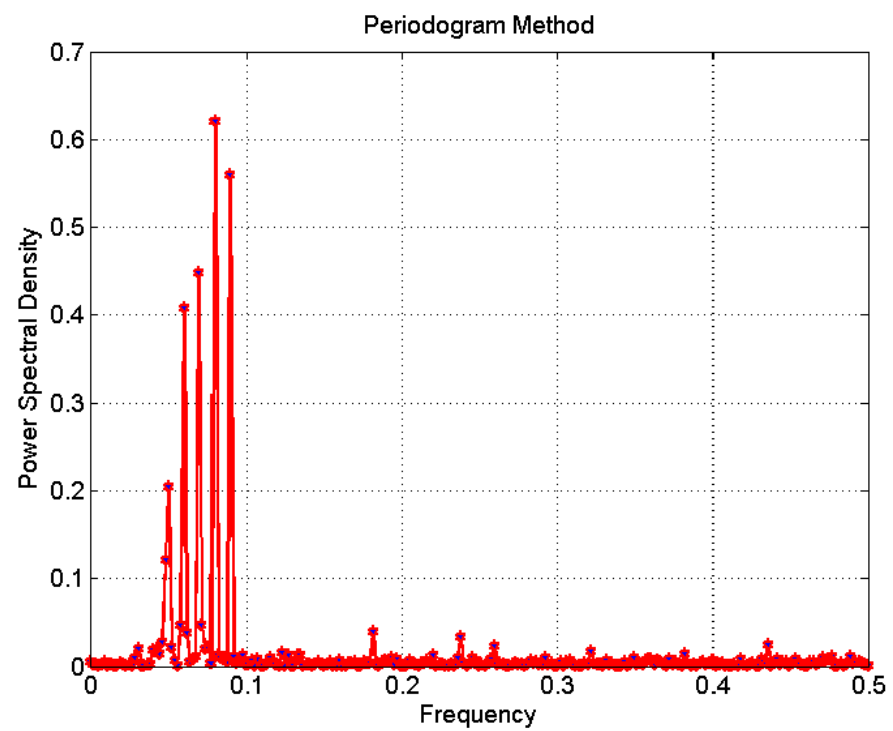

Figure 3. Power Spectral Density of Periodogram Method

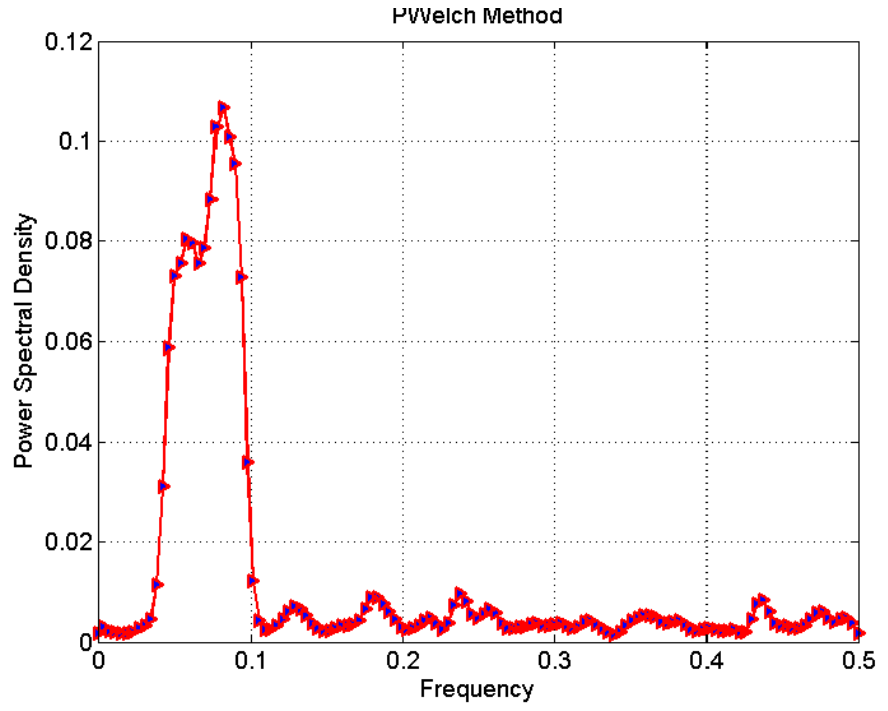

Figure 4. Power Spectral Density of PWelch Method 


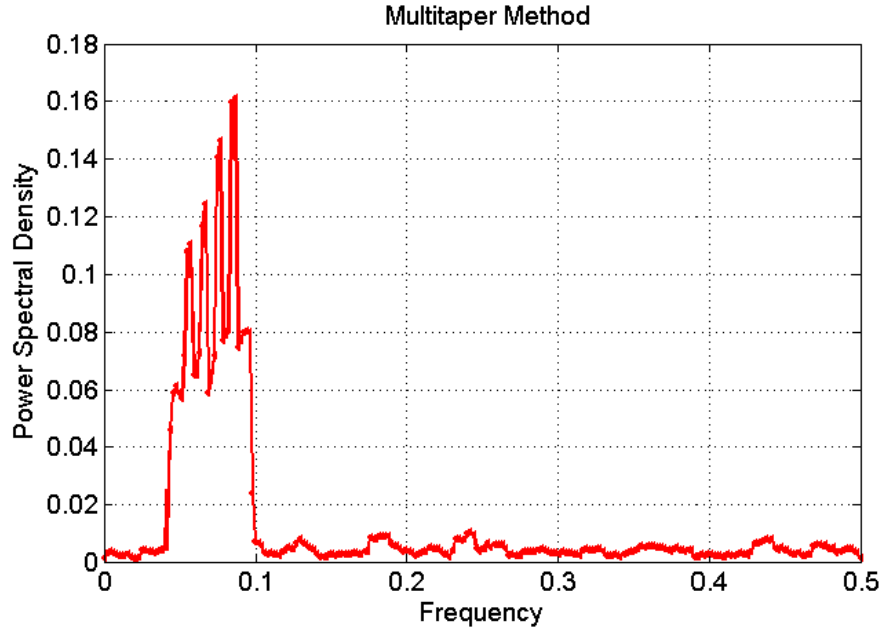

Figure 5. Power Spectral Density of Multitaper Method

The outcome of PSD for Periodogram, PWelch, and Multitaper method shows the spectrum could not exceed more than $0.1 \mathrm{Watt} / \mathrm{Hz}$ of PSD within the range of $0.1-0.5 \mathrm{~Hz}$ of frequency.

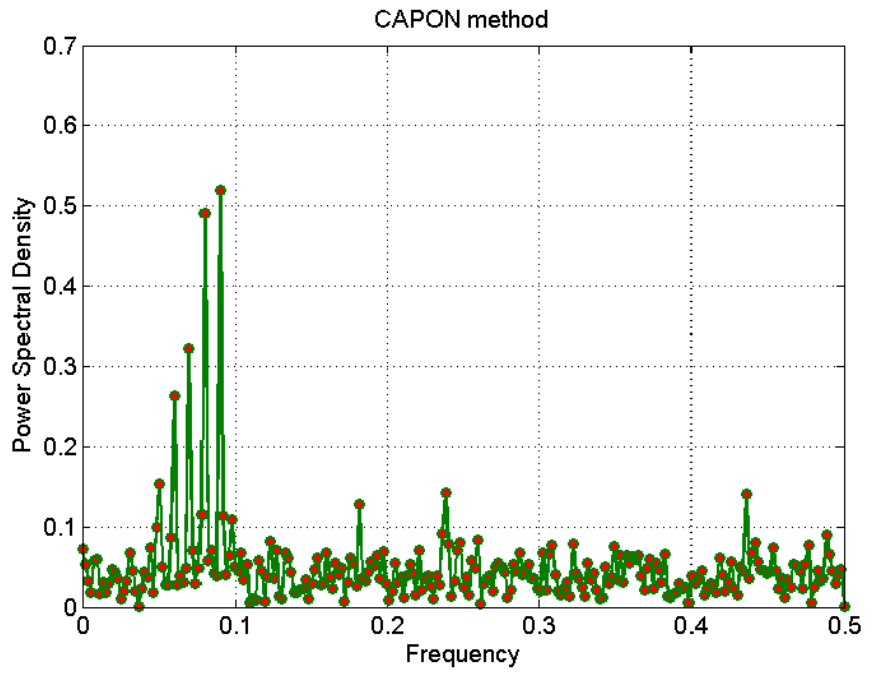

Figure 6. Power Spectral Density of Capon Method 


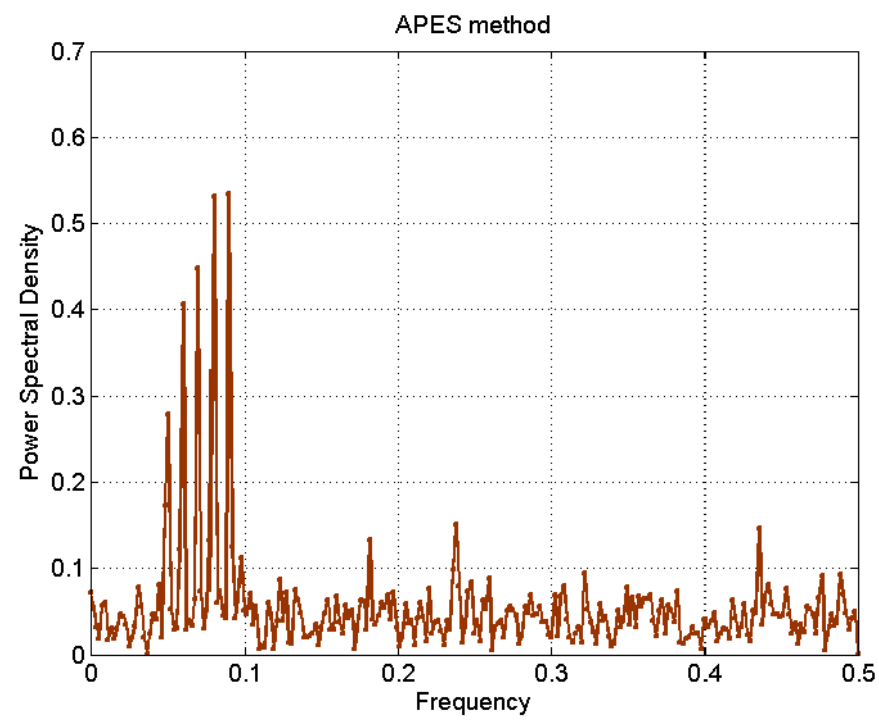

Figure 7. Power Spectral Density of APES Method

Figure 6 and Figure 7 shows the PSD for the Capon and APES method of spectrum estimations. The outcome shows some enhanced values of PSD for both the method as compared to the existing system of Periodogram, PWelch, and Multitaper method.

\section{CONCLUSION}

This paper has attempted to signify that spectral estimation and ensuring its effectiveness plays a critical role in many applications of signal processing. As there are various methods for performing spectral estimations, hence, it is critical to understand the best method to do so. From the literature, we have seen that there are various methods and majority of the researchers are found to be more inclined on Capon and APES estimation techniques. We have also identified a problem in implementation of Capon and APES estimation techniques with respect to computational needs. Hence, this paper has performed an evaluation of the problem associated with various spectral estimation techniques and proposed a simple solution to enhance the power spectral density as well as correlational factor as a mean to showcase the proposed enhanced version of Capon and APES estimation techniques. Our study shows that correlation factor is one of the widely adopted techniques to find the significant relationship among various signals. We also found that essential principle of Capon is not totally used for carrying out spectral analysis. The outcome of our study shows that a CAPON estimator incorporates more data in its signal while APES optimal resolution of the frequencies present in one spectrum. However, we also found certain pitfall in the outcome which shows the overestimation charecteristics of CAPON on minimal amplitude values. Therefore, we will like to solve this problem in future using enhanced version of Recursive Least Square techniques. Our future direction of the study will be focused on achieving further low complexities in computation with efficient speed of convergence for our next enhanced version of Recursive Least Square techniques targeting the time-varying frequencies with impulse components in the signals.

\section{REFERENCES}

[1] P. Golland, N. Hata, C. Barillot, J. Hornegger, R., "Medical Image Computing and Computer-Assisted Intervention", Springer International Conference, Boston, 2014.

[2] M. Subhedar and G. Birajdar, "Spectrum sensing techniques in cognitive radio networks: A Survey", International Journal of Next-Generation Networks, Vol. 3, No. 2, June 2011.

[3] H. Sun, A. Nallanathan, C.X. Wang, "Wideband spectrum sensing for cognitive radio networks: A Survey", IEEE Wireless Communications, 2013.

[4] J. Yang, X. Ma, C. Hou, "Shrinkage-Based Capon and APES for Spectral Estimation", IEEE signal processing letters, vol. 16, no. 10, October 2009.

[5] J.K. Nielsen, P. Smaragdis, M.G. Christensen, "An amplitude spectral capon estimator with a variable filter length", IEEE-Proceedings of the 20th European Signal Processing Conference, pp. 430-434, 2012.

[6] S.R. Alty, A. Jakobsson, and E.G. Larsson, "Efficient implementation of the time-recursive capon and apes spectral estimators", IEEE- European Signal Processing Conference, pp. 1269-1272, 2004. 
[7] K. Angelopoulos, G.O. Glentis, and A. Jakobsson, "Computationally Efficient Capon- and APES-based Coherence Spectrum Estimation", IEEE Transactions of Signal Processing, 2012.

[8] M. Xue, L. Xu, and J. Li, "IAA Spectral Estimation: Fast Implementation Using the Gohberg-Semencul Factorization", IEEE International Conference on Acoustics, Speech and Signal Processing, pp. 4304-4307, 2011.

[9] N.S. Tezel, I. Yıldırım, "Inverse Synthetic Aperture Radar Imaging For Point Scatterers Using APES Algorithm", International Conference on Electrical and Electronics Engineering, 2003.

[10] D. Dan, J. Gong, and Y. Zhao, "Damped CAPES 2D Spectral Estimation for Real-Valued Vibration Signals", Hindawi Publishing Corporation Shock and Vibration, Article ID 570347, 11 pages, 2014.

[11] B. Sridhar, T.Srinivasulu, "A Novel High Resolution Spectrum Sensing Algorithm for Cognitive Radio Applications", IOSR Journal of Electronics and Communication Engineering, Vol. 8, Iss. 4, pp 30-38, 2013.

[12] G.K. Kalognomos, G.E. Boultadakis, A.V. Karakasiliotis, P.V. Frangos, "Performance Analysis of a Parameterized APES (PAPES) Spectrum Estimation Method for ISAR Applications", High frequency technology, microwaves, No. 3, Iss. 99, 2010.

[13] G.L. Touze, B. Nicolas, J.I. Mars, "Double-Capon and double-MUSICAL for arrival separation and observable estimation in an acoustic waveguide", EURASIP Journal on Advances in Signal Processing, 2012.

[14] E.J. Candes, C.A.S. Long, J.D. Trzasko, "Unbiased Risk Estimates for Singular Value Thresholding and Spectral Estimators", IEEE transactions on signal processing, vol. 61, no. 19, October 1, 2013.

\section{BIOGRAPHIES OF AUTHORS}

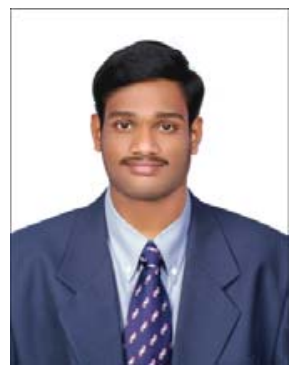

Kantipudi MVV Prasad received his B.Tech degree in Electronics \& communications Engineering from ASR College of Engineering, Tanuku, India, the M.Tech degree in Digital Electronics and Communication Systems from Godavari Institute of Engineering \& Technology, Rajahmundry, India. Currently pursuing his Ph.D from BITS, VTU and working as Assistant Professor in Department of Electronics \& communications, RK University. Rajkot, having teaching experience around 6 years. He has authored and co-authored many papers in International Journals, International Conferences and National Conferences.

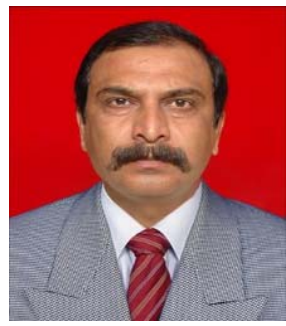

I have received my BE $(E \& C)$ and M.Tech (Bio Medical Instrumentation) from University of Mysore. I got my Ph.D (ECE) from Anna university of Technology. Coimbotore (TN). I had the opportunity to serving as a Chairman for Board of Examiners, and Board of Studies etc. for Visveswaraya Technical University and Bangalore University Karnataka. I worked as Lecturer, Associate professor and Professor and Research / PG co-ordinator in Bangalore institute of technology, Bangalore, affiliated to visvewaraya technological university, Karnataka during 1989 to till date. Presently, I am a Chairman of the Board of examination (IT/ML/BMI) for Visveswaraya technological university, With more than 30 years of academic research and administrative experience blended with progressive views, organizational strengths. I am a member of IEEE, Bio Medical Society of India, ISTE, IMAPS \& Fellow member of IETE. 